\title{
CARI'10
}

\section{Evaluation objective des méthodes de segmentation des maillages polygonaux 3D basée sur la classification de régions}

\author{
Amira Zguira ${ }^{1}$, Narjes Doggaz ${ }^{2}$, Ezzeddine Zagrouba ${ }^{3}$ \\ Equipe de recherche en imagerie intelligente et vision par ordinateur \\ Laboratoire RIADI, Université La Manouba \\ La Manouba 2010, Tunisie \\ ${ }^{1}$ Amira-zguira@hotmail.com, ${ }^{2}$ narjes.doggaz@fst.rnu.tn, ${ }^{3}$ ezzeddine.zagrouba@fsm.rnu.tn

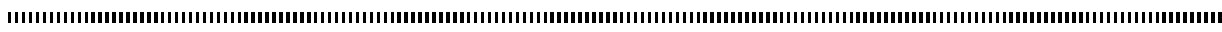

RÉSUMÉ. Dans ce papier, nous proposons une approche d'évaluation objective des algorithmes de segmentation des maillages polygonaux 3D, basée sur la classification de régions. Cette approche commence par un processus de classification de régions du maillage constituant la vérité-terrain en régions convexes, concaves et planes. Nous présentons ensuite trois mesures de qualité qui permettent de quantifier la similarité de chaque type de région de la vérité-terrain par rapport à la segmentation obtenue par un algorithme automatique. Nous appliquons l'approche proposée sur un échantillon de huit techniques de segmentation bien choisies sur une base d'images hétérogène. Les résultats d'évaluation obtenus permettent d'assurer une meilleure compréhension des points forts et faibles de chaque technique dans la segmentation des différents types de régions du maillage et ceci afin d'aiguiller correctement le choix des algorithmes de segmentation pour différentes applications.

ABSTRACT. In this paper, we propose an objective evaluation approach of polygonal 3D mesh segmentation algorithms. Our approach is based on region classification. For that, we classify first manual segmented mesh into convex, concave and planar regions. Secondly, we present three quality measures that quantify the similarity of each type of region of the ground-truth relatively to the segmentation obtained by an automatic algorithm. We apply this approach on eight wellselected existing algorithms on heterogeneous images. This provides better understanding as to the strengths and weaknesses of each technique in function of each mesh-regions type in the aim to make the better choice concerning the segmentation algorithms for different applications.

MOTS-CLÉS : maillage 3D, type de région, segmentation, évaluation, vérité-terrain.

KEYWORDS: 3D mesh, region type, segmentation, evaluation, ground-truth. 


\section{Introduction}

La représentation tridimensionnelle (3D) d'un objet tient actuellement une place importante dans les systèmes multimédias et s'avère très utile dans des applications de plus en plus nombreuses (la cartographie, la simulation, la conception assistée par ordinateur, les jeux vidéo, le cinéma, la modélisation d'environnements réels ou virtuels, etc.). Les maillages polygonaux 3D occupent une position primordiale parmi les différents modes de représentation informatique d'objets géométriques et constituent un de ces modes les plus répandus.

Motivés par la complexité de mise en œuvre de la segmentation de maillages 3D et la multitude d'applications qui en sont dépendantes, beaucoup de chercheurs se sont intéressés à ce domaine de recherche. La segmentation de maillages polygonaux 3D consiste à subdiviser une surface 3D en un ensemble de régions différentes présentant des caractéristiques similaires, soient géométriques (segmentation en patches surfaciques) ou sémantiques (segmentation en parties significatives) [6]. Une multitude de méthodes de segmentation des maillages 3D ont été développées et elles sont basées sur des approches différentes. Vu le nombre considérable et la variété de ces méthodes, le problème de l'évaluation de la qualité de la segmentation s'avère primordial. En effet, il est nécessaire d'effectuer une évaluation et une comparaison objective des méthodes existantes afin de juger de leur efficacité et de leur pertinence. Jusqu'à présent, peu de chercheurs ont abordé ce problème. Certains travaux ont proposé des modèles de véritéterrain et des mesures quantitatives d'évaluation objective généralisées à partir des mesures d'évaluation de segmentation des images 2D [4] [5] [6] [7]. Cependant, ces mesures évaluent seulement la qualité des résultats obtenus pour l'ensemble de l'image toute entière et elles ne peuvent pas être adaptées à l'évaluation de la performance d'une méthode de segmentation par rapport au type de régions segmentées du maillage.

Juger objectivement la qualité de la segmentation pour chaque type de région est la motivation principale de ce papier. Nous proposons trois métriques pour mesurer la similarité pour chaque type de régions segmentées. Ces métriques sont inspirées de l'évaluation de segmentation des images 2D [1]. Notre approche d'évaluation est basée sur un processus de classification en régions convexes, concaves et planes. Nous appliquons cette approche sur huit algorithmes en utilisant une vérité-terrain relative à une base d'images hétérogènes [7]. Ce papier est organisé comme suit. La section 2 présente un état de l'art de l'évaluation de la segmentation de maillages 3D. Nous présentons le principe de l'approche proposée dans la section 3. Dans la section 4, nous décrivons le processus de classification des régions segmentées. Puis, en section 5 , nous présentons les trois métriques objectives proposées pour chaque type de région. La section 6 met en valeur ces métriques en les appliquant sur un échantillon de huit algorithmes de segmentation, puis nous analysons les résultats de l'évaluation de ces algorithmes. Enfin, nous concluons. 


\section{Etat de l'art de l'évaluation de la segmentation}

Dans la littérature, Il existe plusieurs travaux concernant le problème de l'évaluation de la qualité de la segmentation des images 2D. De ce fait, de nombreuses méthodes d'évaluation de segmentation d'images ont été proposées au cours des dernières décennies. Ces approches d'évaluation peuvent être classées en deux grandes catégories: l'évaluation supervisée et l'évaluation non supervisée selon que la méthode nécessite ou non une image de référence d'une vérité-terrain [16]. Par analogie à l'évaluation 2D, les critères d'évaluation quantitative en 3D peuvent se regrouper aussi en deux classes selon qu'on dispose ou non d'une vérité-terrain qui constitue une segmentation de référence.

En l'absence de vérité-terrain, des critères quantitatifs absolus ou des calculs de cohérence entre les différents résultats de segmentation peuvent être utilisés. En effet, Attene et al. [3] soulignent la difficulté d'évaluation de la segmentation compte tenu des différents contextes d'utilisation. Ils ont défini plusieurs critères d'évaluation de segmentation ; à savoir : le type de segmentation, la complexité, la sensibilité à la pose, etc. Ces critères abordés sont très importants, mais ils ne permettent pas de valider la pertinence des régions extraites ni de mesurer leur rapprochement par rapport à la perception visuelle humaine. Récemment, Delest et al. [8] ont montré les avantages d'une vérité-terrain pour la segmentation de maillages 3D. En utilisant une vérité-terrain, Benhabiles et al. [6] ont proposé tout d'abord une approche d'évaluation objective des algorithmes de segmentation de maillage 3D. En effet, ils ont généralisé la distance de Hausdorff classique et modifiée. Benhabiles et al. [5] ont ensuite proposé une autre approche d'évaluation objective qui est fondée sur deux mesures de l'erreur de raffinement. Ils ont testé les mesures proposées sur deux algorithmes de segmentation. Les mesures proposées dans [5] et [6] sont basées sur un corpus de vérité-terrain contenant des modèles de différents objets 3D avec leurs segmentations manuelles produites par des experts humains. Cependant, ce corpus contient un ensemble limité de modèles 3D qui sont segmentés manuellement. Dans le même cadre, Chen et al. [7] ont proposé un benchmark qui contient une étude comparative de sept algorithmes. Ils ont testé ces algorithmes sur une grande base de modèles de maillages 3D. Ils ont introduit un ensemble de critères d'évaluation quantitatifs. Certaines de ces métriques ont été comparées dans un récent travail de Benhabiles et al. [4] selon des propriétés, en proposant une nouvelle mesure de similarité appelée l'Indice de Rand Probabiliste Normalisé pour la 3D (3D-NPRI). Ils ont montré que cette nouvelle métrique donne de meilleurs résultats que les autres en terme de propriétés (non-dégénérescence, indépendance de la cardinalité, tolérance au raffinement, vérités-terrains multiples, comparaison significative) et de pouvoir discriminant. Cependant, les critères proposés dans ces travaux tiennent compte d'une évaluation globale du maillage. Ces critères permettent en effet d'évaluer seulement la qualité des résultats obtenus pour une image donnée sans tenir compte de l'évaluation par rapport à chaque type de région segmentée. 
Notre travail offre une analyse de la qualité de segmentation par rapport à chaque type de régions segmentées (convexe, concave ou plane). Cette évaluation fournit une meilleure compréhension de l'utilisation de certains critères lors du processus de segmentation du maillage. En outre, elle mesure la performance d'une méthode de segmentation relativement au type de régions segmentées d'un objet 3D.

\section{Principe général de l'approche proposée}

Nous proposons une nouvelle approche d'évaluation en s'inspirant de ce qui a été effectué en évaluation de segmentation d'images 2D [1]. En effet, dans ce travail, Amri et Zagrouba [1] ont effectué une étude comparative quantitative de sept méthodes de segmentation d'images-2D en régions. Pour ce faire, ils ont proposé deux nouvelles mesures d'évaluation de segmentation en régions en utilisant la mesure de Vinet tout en étant guidé par une classification en zones uniformes et texturées. Ils ont utilisé la fonction de luminance comme caractéristique principale pour classifier les régions segmentées. Par analogie, nous proposons d'utiliser la courbure de la surface comme caractéristique principale autour de laquelle le processus de classification sera réalisé.

Dans le cadre de notre approche, nous commençons par classifier le maillage de la vérité-terrain en trois types de régions : à savoir les régions convexes, concaves et planes. Cette classification est basée sur le calcul de courbures principales de chaque

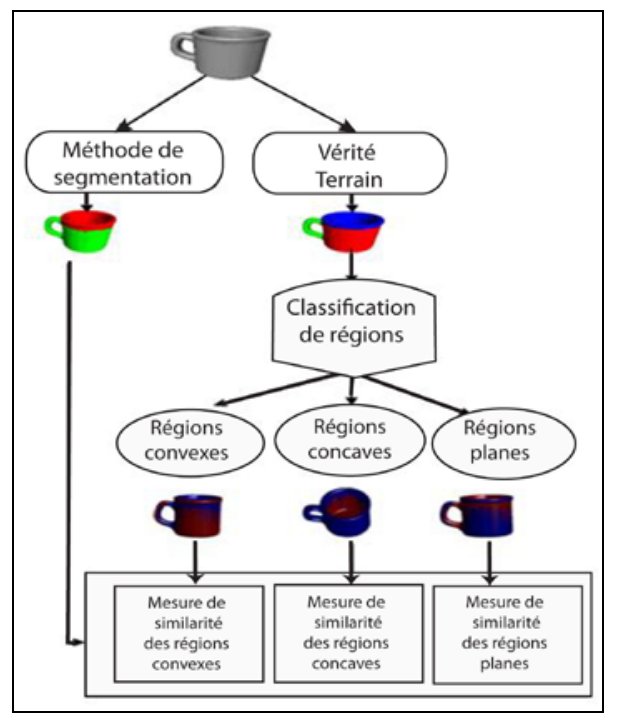

Figure 1. Schéma global de l'approche proposée. 
région. Par la suite, une évaluation de la qualité de la segmentation automatique pour les différents types de régions est effectuée. Pour ce faire, trois mesures de similarité sont proposées. Chaque mesure est relative à un type de région (figure 1).

\section{Classification des régions segmentées}

L'approche proposée commence par le processus de classification en régions du maillage de référence segmenté. Cette étape est très importante dans notre approche afin d'évaluer les résultats de deux segmentations par rapport à chaque nature de région. L’identification du type de chaque région de l'image référence a été réalisée en utilisant le calcul des courbures gaussiennes et moyennes et selon chaque valeur calculée pour une région, nous pouvons distinguer entre les régions convexes, concaves et planes. En effet, il existe dans la littérature plusieurs méthodes pour classifier les différentes régions d'un maillage $3 \mathrm{D}$. Ces méthodes sont basées sur les différentes propriétés topologiques et géométriques du maillage. Nous avons opté pour la classification des régions en fonction des valeurs de courbures moyennes et gaussiennes, vue sa précision, sa consistance et la simplicité de sa mise en œuvre. Pour ce faire, nous avons appliqué l'approche proposée par Meyer et al. [13], en utilisant la moyenne de cellule de Voronoï et la méthode mixte d'élément fini/volume fini (figure 2). En effet, leur approche consiste à sélectionner un élément fini linéaire sur chaque triangle du maillage. Cet élément constitue une interpolation linéaire entre les trois sommets correspondant à chaque triangle. Pour chaque sommet sélectionné, une surface associée patch (appelée volume fini) est choisie, sur laquelle la moyenne est calculée.

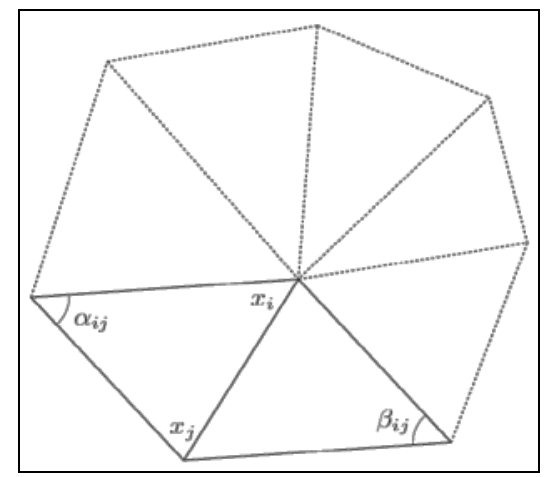

Figure 2. 1-voisinage du vertex $x_{i}$ et les angles opposés à une arête [13]. 
La courbure moyenne $C_{m}$ est calculée avec l'équation suivante [14] :

$$
C_{m}\left(x_{i}\right)=\frac{1}{2 A} \sum_{j \in N_{1}(i)}\left(\cot \alpha_{i j}+\cot \beta_{i j}\right)\left(x_{i}-x_{j}\right)
$$

Où A est la surface de la zone de Voronoi au point $X_{i}$ :

$$
A=\frac{1}{8} \sum_{j \in N_{1}(i)}\left(\cot \alpha_{i j}+\cot \beta_{i j}\right)\left\|x_{i}-x_{j}\right\|^{2}
$$

Où $\alpha_{i j}$ et $\beta_{i j}$ sont les deux angles opposés au coté $\left(X_{i}, X_{j}\right)$ et $N_{1}(i)$ est le 1voisinage du point $X_{i}$.

La courbure gaussienne $C_{g}$ est donnée par :

$$
C_{g}\left(x_{i}\right)=\frac{1}{A}\left(2 \pi-\sum_{j=1}^{f} \theta_{j}\right)
$$

Où $f$ correspond au nombre de triangles du 1 -voisinage du point $X_{i}$ et $\theta_{j}$ correspond à l'angle du triangle $j$ en $X_{i}$.

Après avoir estimé les valeurs de courbures des sommets d'une région d'une image segmentée, nous calculons les courbures de toutes les régions de la même image et puis, nous répétons ce calcul pour tous les modèles segmentés des images de la référence. Les valeurs des courbures moyennes et gaussiennes peuvent être positives, négatives ou nulles. Selon les valeurs de ces courbures, nous classifions le maillage en trois zones différentes : zone convexe, zone concave et zone plane. La première classe de régions présente les régions convexes ayant une courbure moyenne positive et une courbure gaussienne positive (figure 3a). La deuxième présente les régions concaves possédant une courbure moyenne négative et une courbure gaussienne positive (figure $3 \mathrm{~b}$ ). $\mathrm{La}$ dernière classe est la classe des régions planes ayant une courbure gaussienne presque nulle ou négative (figure 3c). Nous faisons remarquer que dans la figure 3 les régions convexes, concaves et planes sont colorées en rouge. 


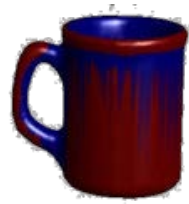

(a) Régions convexes

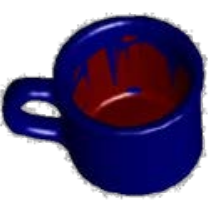

(b) Régions concaves

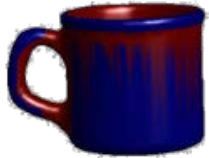

(c) Régions planes

Figure 3. La classification des régions convexes, concaves et planes du modèle Cup.

\section{Mesures d'évaluation relatives au type de région}

L'étape présente de notre approche est de développer une métrique d'évaluation quantitative objective. Pour ce faire, nous définissons trois mesures. Chaque mesure est relative à un type de région (convexe, concave ou plane). Chaque région du maillage segmenté est, à ce stade, assortie de sa nature (convexe, concave ou plane).

Etant donné un ensemble $I$ de $n$ images, $I=\left\{I_{k} / 1 \leq k \leq n\right\}$, on associe à chaque image $I_{k}: \operatorname{Seg}_{k}=\left\{R_{k}^{1} \ldots R_{k}^{N_{k}}\right\}$ l'ensemble de régions segmentées automatiquement et $R e f_{k}=\left\{r_{k}^{1} \ldots r_{k}^{M_{k}}\right\}$ l'ensemble de régions segmentées manuellement. Avec $N$ le nombre de régions du maillage segmenté automatiquement et $M$ le nombre de régions du maillage segmenté manuellement.

Après la phase d'identification du type de chaque région de l'image de référence $R e f_{k}$, nous obtenons trois ensembles :

$$
\begin{aligned}
& R e f_{k}^{c n v}=\left\{r_{k}^{j} / 1 \leq j \leq M_{k} \text { et } r_{k}^{j} \text { convexe }\right\} \\
& \operatorname{Re} f_{k}^{c n c}=\left\{r_{k}^{j} / 1 \leq j \leq M_{k} \text { et } r_{k}^{j} \text { concave }\right\} \\
& \operatorname{Re}_{k}^{p \ln }=\left\{r_{k}^{j} / 1 \leq j \leq M_{k} \text { et } r_{k}^{j} \text { plane }\right\}
\end{aligned}
$$

Avec : $R e f_{k}=R e f_{k}^{c n v} \cup R e f_{k}^{c n c} \cup R e f_{k}^{p \ln }$.

Etant donné $R_{k}^{i}$ (respectivement $r_{k}^{j}$ ) l'ensemble des sommets constituant la $i$ ème région de l'image $k$ segmentée automatiquement (respectivement la $j$ ème région de 
l'image $k$ segmentée manuellement), pour chaque image $I_{k}$ de la base, on définit une table de similarité $T_{k}$ où chaque élément est défini par :

$$
T_{k}(i, j)=\frac{1}{2}\left(\frac{\operatorname{card}\left(R_{k}^{i} \cap r_{k}^{j}\right)}{\operatorname{card}\left(R_{k}^{i}\right)}+\frac{\operatorname{card}\left(R_{k}^{i} \cap r_{k}^{j}\right)}{\operatorname{card}\left(r_{k}^{j}\right)}\right)
$$

Chaque élément de la table constitue la moyenne arithmétique de deux ratios qui sont le nombre de sommets communs entre les deux régions $R_{k}^{i}$ et $r_{k}^{j}$ par rapport à la cardinalité de la segmentation automatique et par rapport à la cardinalité de la segmentation de référence. Ensuite, nous définissons trois mesures de similarité associées respectivement aux régions convexes, concaves et planes (eq 8). Chacune de ces mesures calcule le recouvrement maximal par rapport à chaque type de région pour toutes les images de la base.

$$
Z_{\text {type }}^{n}=\frac{\sum_{k=1}^{n}\left(\sum_{j=1 / r_{k}^{j} \in \operatorname{Re} f_{k}^{t y p e}}^{M_{k}} \max _{1 \leq i \leq N_{k}}\left(T_{k}(i, j)\right)\right)}{\sum_{k=1}^{n} M_{k}^{\text {type }}}, \text { type }=\{c n v, \text { cnc }, p \ln \}
$$

Cette métrique d'évaluation qui est relative à chaque type de région est une nouvelle métrique pour l'évaluation quantitative des méthodes de segmentation des maillages 3D, inspirée de ce qui a été effectué en évaluation de segmentation d'images 2D [1]. En effet, l'aspect des régions convexes, concaves et planes s'avère être un critère important pour séparer les principales caractéristiques d'un maillage. Evaluer la qualité de segmentation en fonction des caractéristiques géométriques des régions permet une comparaison plus objective entre les méthodes de segmentation.

\section{Etude expérimentale}

Pour valider notre approche d'évaluation, nous avons sélectionné une base de 42 images hétérogènes (figure 4) avec leurs vérité-terrain (figure 5) et nous l'avons appliquée sur huit techniques de segmentation (TAB.1). 

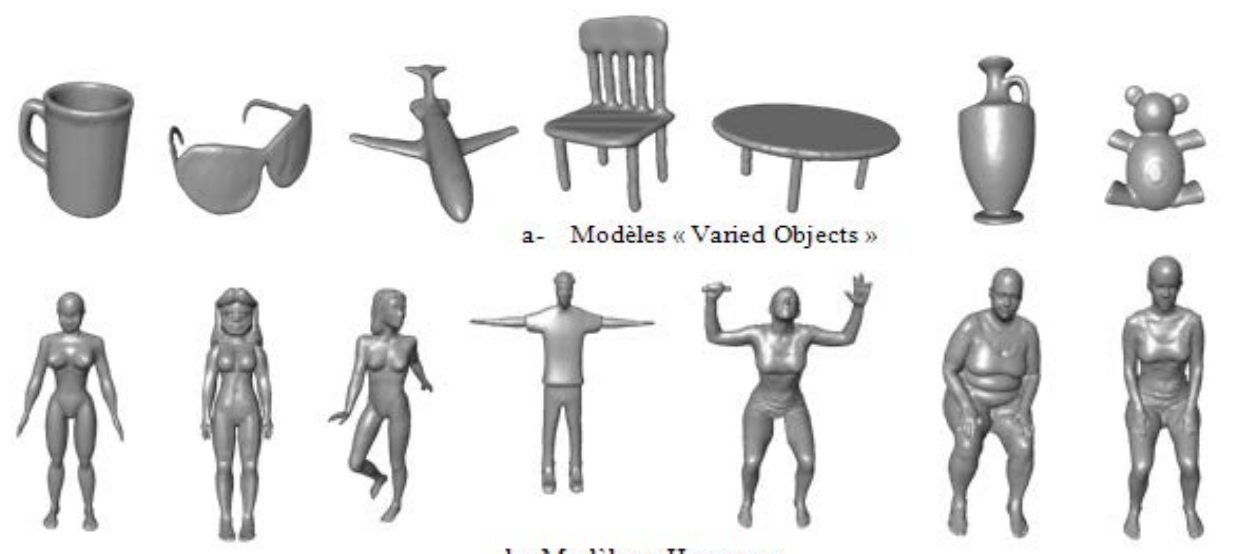

b- Modèles « Human *

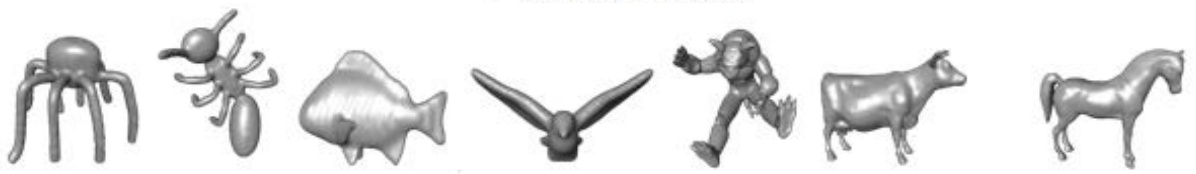

c- Modèle "Animals "

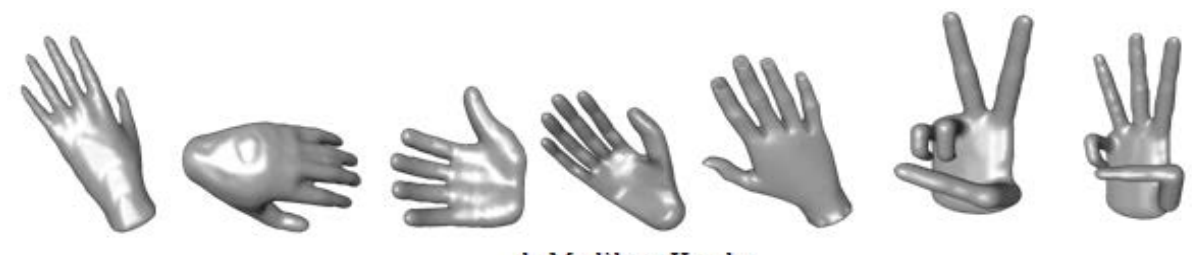

d- Modèles « Hand"

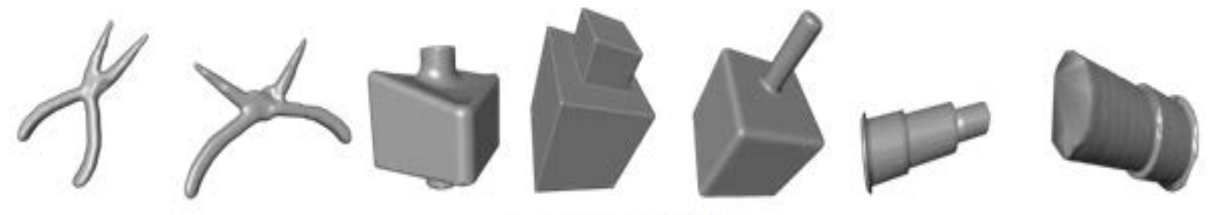

e- Modèles «CAD *
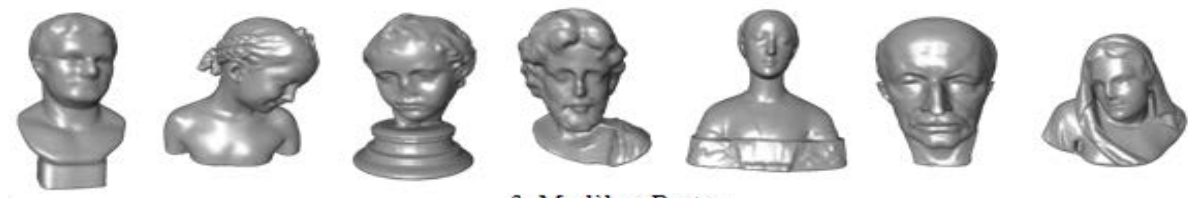

f- Modèle « Bust »

Figure 4. Les différents modèles de notre base d'images [7]. 
Les images sur lesquelles nous avons testé notre approche proviennent de la base d'images du benchmark ${ }^{1}$ de Chen et al. [7].

La vérité-terrain a été obtenue en demandant à un groupe d'experts de segmenter manuellement les maillages en parties fonctionnelles et en leur fournissant un ensemble d'outils dont l'interface de coloriage d'objet 3D. La tâche de segmentation manuelle a été effectuée librement sans dicter aucune condition à l'expert [7]. Pour notre évaluation nous avons sélectionné les vérités-terrain qui nous ont semblé les plus sémantiques. Dans la figure 5, nous présentons quelques modèles de vérité-terrain que nous avons sélectionnés.

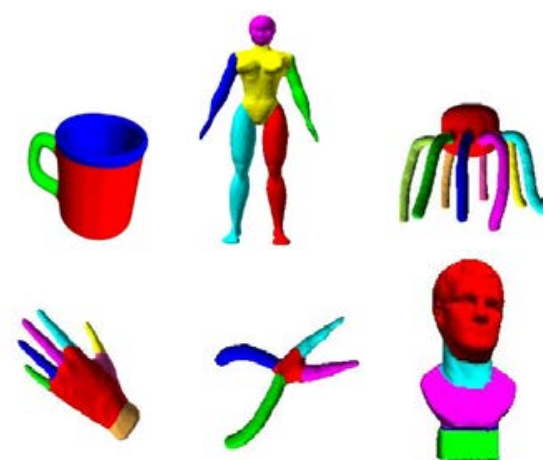

Figure 5. Quelques modèles de la vérité-terrain [7].

Le tableau 1 présente les huit méthodes de segmentation retenues et leurs notations. Nous avons spécifié aussi le type de segmentation pour chaque méthode (parties significatives ou patches surfaciques). Pour effectuer notre choix, nous nous sommes surtout focalisés sur des travaux récents. Par ailleurs, nous avons privilégié les approches qui adoptent une segmentation sémantique K-Median, Core Extra, Norm Cuts, Rand Cuts, Shape Diam et Rand Walks.

Ainsi, ces méthodes peuvent être regroupées en deux classes : les méthodes utilisant des propriétés locales de la forme (K-Median, RG, Rand Walks et Fit Prim) et les méthodes utilisant des propriétés non-locales de la forme (Shape Diam, Core Extra, Norm Cuts et Rand Cuts).

\footnotetext{
${ }^{1}$ http://segeval.cs.princeton.edu/
} 


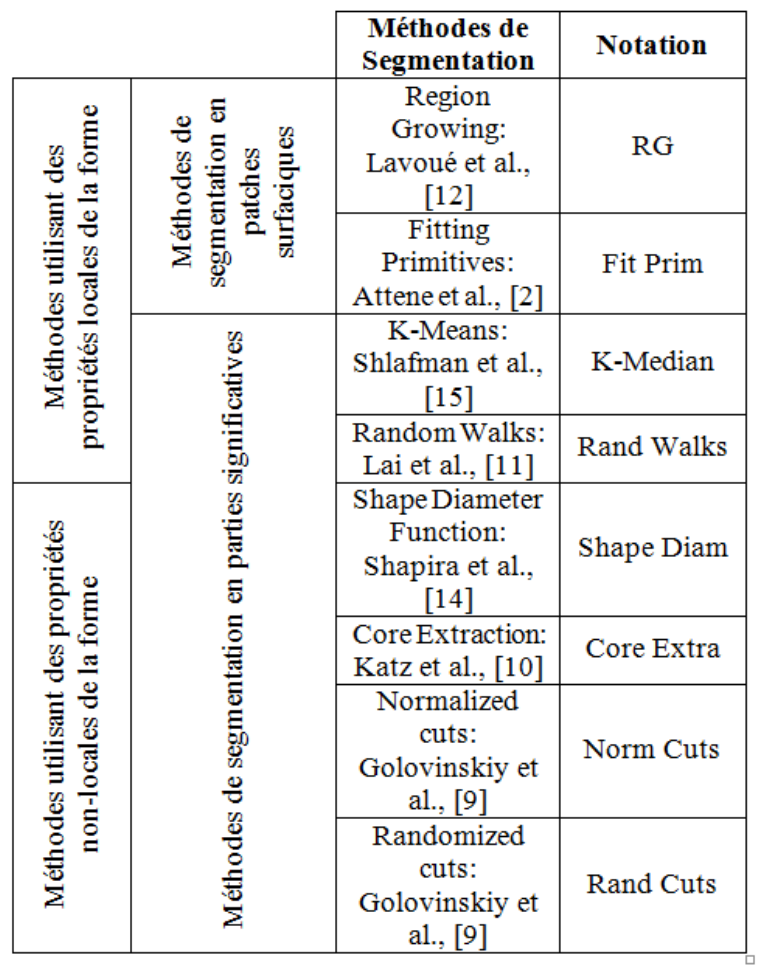

TAB. 1. Les méthodes de segmentation retenues.

Dans le premier groupe, la forme de patchs extraits est guidée par des contraintes de paramétrisation, de planéité, de courbure, etc. De ce fait, les algorithmes présentés dans [12], [15], [11], [2] appartiennent au premier groupe de méthodes qui considèrent l'utilisation des propriétés locales de la forme. Par exemple, Lavoué et al. [12] utilisent les valeurs de la courbure comme critère dans son algorithme de croissance de région. La segmentation hiérarchique proposée par Attene et al. [2] est basée sur le calcul du meilleur ajustement par rapport à des primitives (plan, cylindre ou sphère) pour approximer les faces du maillage à segmenter.

La figure 6 illustre les résultats de segmentation des deux modèles de maillages produits par les algorithmes de segmentation du premier groupe. 

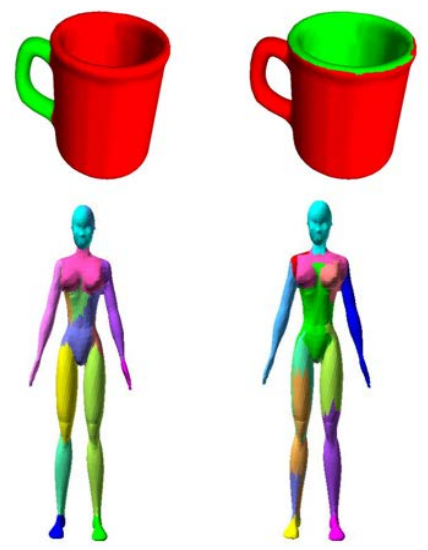
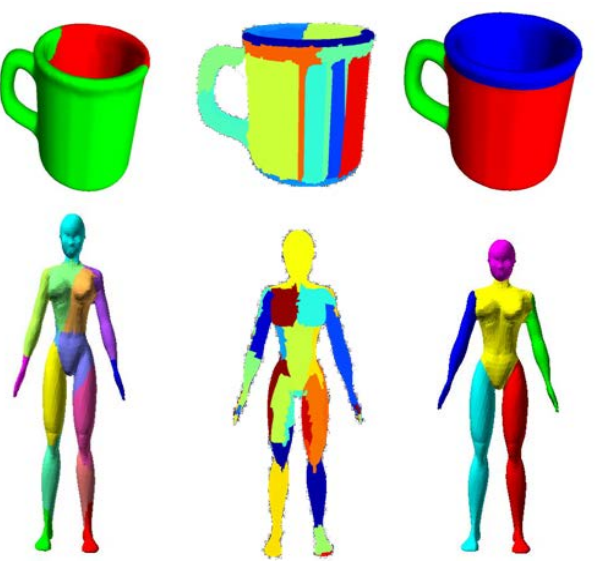

Figure 6. Les résultats de segmentation des modèles cup et human. De gauche à droite : Rand Walks, Fit Prim, K-Median, RG et la vérité-terrain.

L’image correspondante à la vérité-terrain du modèle cup est segmentée en trois parties (convexe, concave et plane). Nous remarquons qu'il y a une différence entre le les résultats des méthodes de segmentation en terme de similarité par rapport à la véritéterrain (figure 6). Ces résultats montrent que la méthode Rand Walks est l'une des bonnes méthodes, de ce premier groupe, qui segmente les régions convexes et concaves. Par contre la méthode RG s'avère la moins bonne en segmentant les régions convexes du modèle cup. Elle génère une sur-segmentation. Pour les régions planes des deux modèles cup et human, nous remarquons une bonne adaptation de la méthode Fit Prim en segmentant ce type de région.

Concernant le second groupe, il constitue les méthodes qui tiennent compte des propriétés non-locales de la forme dans le processus de segmentation. Par exemple, Shapira et al. [14] et Katz et al. [10] utilisent les propriétés des objets volumétriques dans le voisinage d'un point. Les méthodes Randomized-cuts [9] et Normalized-cuts [9] sont basées sur des fonctions objectives coûteuses qui utilisent des propriétés à grande échelle des frontières des segments.

La figure 7 montre les résultats de segmentation des mêmes modèles cup et human produits par les méthodes de segmentation automatiques de ce deuxième groupe et la vérité-terrain. Nous pouvons constater visuellement que les images correspondant aux résultats de segmentation par les méthodes qui adoptent les propriétés non-locales de la forme sont plus proches de la vérité-terrain que les segmentations effectuées par les méthodes utilisant des propriétés locales de la forme. 

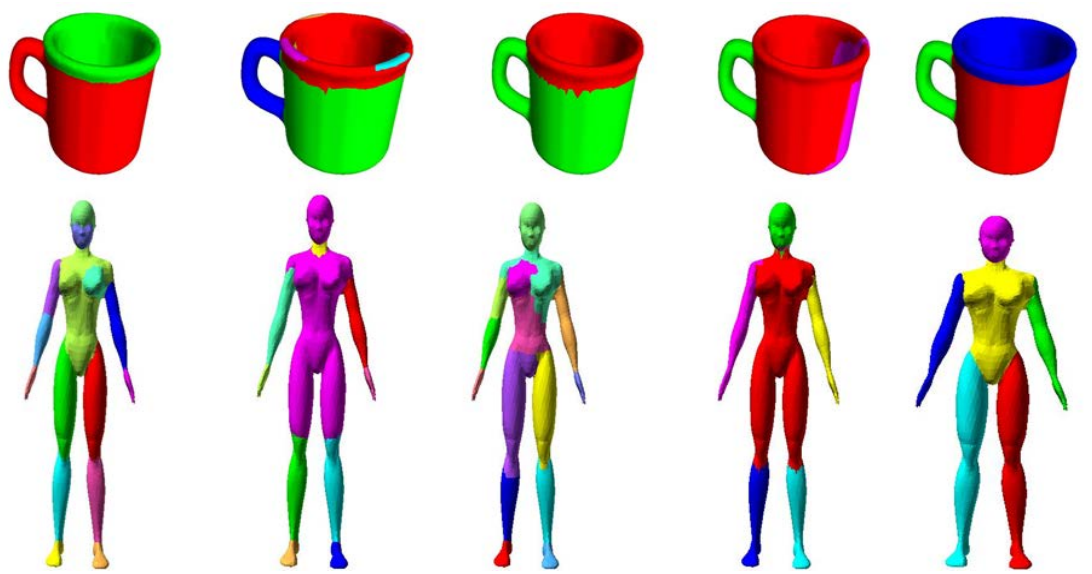

Figure 7. Les résultats de segmentation des modèles cup et human. De gauche à droite : Rand Cuts, Shape Diam, Norm Cuts, Core Extra et la vérité-terrain.

La figure 8 présente les résultats expérimentaux de l'application de notre métrique pour l'évaluation de la segmentation des maillages 3D pour le modèle cup (eq 8). Nous présentons ainsi dans le même graphique, la performance de chaque technique de segmentation dans les trois types de régions.

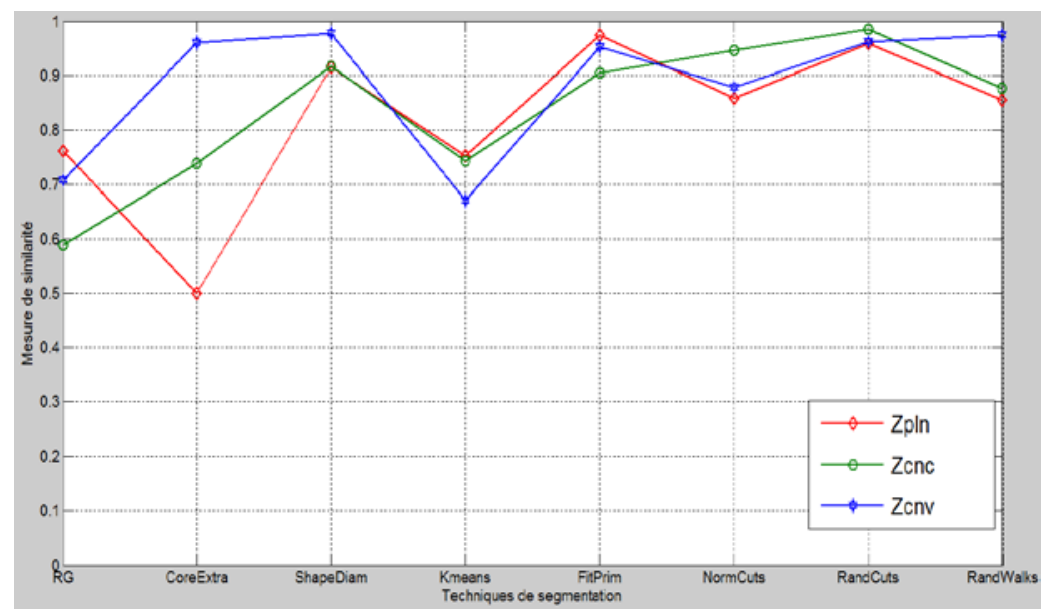

Figure 8. Evaluation selon notre mesure de similarité des méthodes de segmentation sur la base du modèle Cup. 
Les valeurs portées sur le graphique ci-dessus (figure 8) sont celles de la mesure de similarité, pour les huit méthodes de segmentation, des régions convexes (en bleu), concaves (en vert) et planes (en rouge). Les méthodes sont placées sur l'axe des abscisses. Ça nous permet de déduire la variation de ces trois mesures dans les différentes méthodes de segmentation. Une grande valeur de mesure de similarité de région (plus proche de 1 ) correspond à la meilleure méthode de segmentation dans les régions de même nature.

Nous remarquons d'après le graphique de la figure 8 que les méthodes Rand Walks, Core Extra, Shape Diam et Rand Cuts sont les mieux adaptées pour segmenter les régions convexes du modèle cup.

Les méthodes Rand Cuts et Norm Cuts sont les méthodes les plus appropriées à segmenter les régions concaves du modèle cup. Cependant, la méthode Fit Prim est la meilleure méthode pour segmenter les régions planes. Nos résultats expérimentaux sont cohérents avec les résultats visuels de segmentation (figures 6 et 7) et cela prouve que notre mesure de similarité évalue bien la qualité de segmentation d'un objet pour les différentes méthodes.

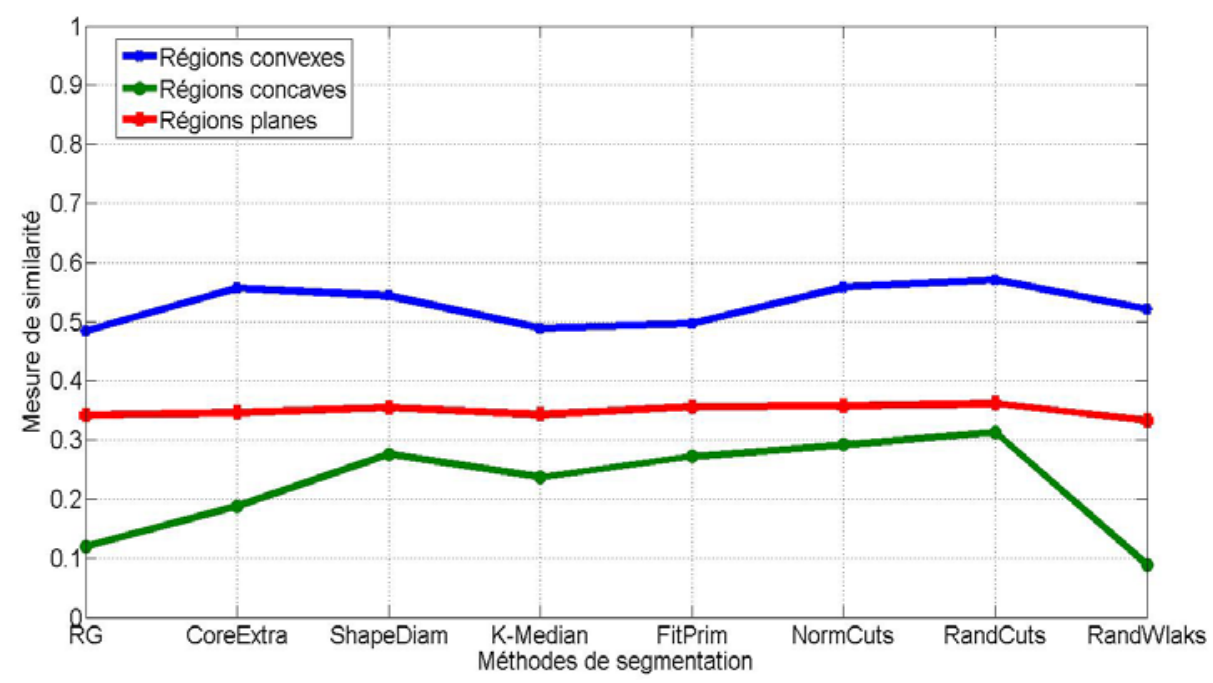

Figure 9. Evaluation relativement aux trois mesures de similarité. 
La figure 9 illustre les résultats de notre mesure de similarité (eq 8) obtenue pour chaque méthode de segmentation pour toutes les images de la base. Pour une méthode de segmentation donnée, une mesure de similarité des régions convexes (respectivement concaves et planes) élevée indique une bonne adéquation de la méthode en question pour la segmentation des régions convexes (respectivement concaves et planes). Nos résultats expérimentaux ont montré que les techniques de segmentation adoptant des propriétés non locales de la forme (Rand Cuts, Norm Cuts, Core Extra et Shape Diam) sont meilleures que celles s'appuyant sur les propriétés locales de la forme. Nous constatons en particulier que Rand Cuts est la meilleure méthode pour segmenter les régions convexes, concaves et planes. Néanmoins, la segmentation par Rand Walks est la moins adaptée pour la segmentation des régions planes et concaves. La méthode RG est cependant la moins bonne pour segmenter les régions convexes.

Les résultats concernant l'évaluation de la segmentation des régions convexes et concaves présentent les mesures de qualité les plus dispersées (variance $_{\mathrm{cnv}}=$ 0.00119277 , variance ${ }_{\mathrm{cnc}}=0.0087033$ ). Cela est expliqué par la variété des formes convexes et concaves qui peuvent être segmentées de façons différentes par les méthodes en utilisant divers critères. Cependant, les méthodes de segmentation des régions planes présentent les mesures de similarité les plus proches (variance $_{\text {pln }}=$ 9.6161E-05). En effet, les zones planes ont la même forme géométrique de manière à être segmentées presque de la même façon et cela permet d'avoir des résultats très proches. Par ailleurs, nos résultats concernant les régions planes montrent la performance de certains algorithmes qui sont fréquemment utilisés en CAO (Conception Assistée par Ordinateur) dans la segmentation de ce type de régions. Par exemple, la méthode Fit Prim, qui est composée de primitives géométriques, telles que les modèles de CAO, est la plus adaptée pour segmenter ce type d'objets [2].

Ainsi, les critères utilisés dans chaque méthode lors de la segmentation ont une influence sur la qualité des régions segmentées. En effet, chaque méthode utilise certains critères pour guider le processus de segmentation où le type des régions extraites dépend des critères adoptés. Par conséquent, à travers la phase de classification établie avant l'application de la métrique d'évaluation, notre approche assure une meilleure compréhension de l'utilisation de ces critères dans le processus de segmentation du maillage. Cela permet alors de fournir une meilleure comparaison des points forts et faibles de chaque technique dans la segmentation de chaque type de régions du maillage à segmenter. C'est pourquoi, nous avons pensé à déterminer le recouvrement maximal sur des zones de l'image de même type et non sur l'image toute entière. En outre, cette approche peut aider dans le choix de l'algorithme de segmentation le plus adapté à chaque zone de l'image 3D et ceci dans des applications telles que : le tatouage, la compression, l’imagerie médicale, etc. 


\section{Conclusion}

Dans ce papier, nous proposons une nouvelle approche d'évaluation objective de méthodes de segmentation des maillages 3D. À cette fin, après avoir choisi une base d'images hétérogènes munie d'une vérité-terrain, nous avons classifié les régions segmentées issues de la vérité-terrain selon le calcul de courbures principales. Après avoir défini le type de chaque région de l'objet (convexe, concave ou plane), nous avons proposé trois mesures de similarité pour l'évaluation de la qualité de segmentation pour chaque type de région. Pour valider l'approche, nous avons sélectionné huit algorithmes de segmentation récents.

Nous projetons d'effectuer davantage d'expérimentations sur des bases de tests plus larges afin d'établir une étude plus complète pour une évaluation objective de la segmentation des maillages 3D.

\section{Bibliographie}

[1] Amri S. et Zagrouba E. (2006). Evaluation and fusion of image segmentation methods. En ICTTA : International Conference on Information \& Communication Technologies : From Theory to Applications, vol. 1, 1524-1529.

[2] Attene M., Falcidieno, B., et Spagnuolo M. (2006). Hierarchical mesh segmentation based on fitting primitives. Vis. Comput., vol. 22(3), 181-193.

[3] Attene M., Katz S., Mortara M., Patan G., Spagnuolo M. et Tal A. (2006). Mesh segmentation, a comparative study. En SMI : Proceedings of the IEEE International Conference on Shape Modeling and Applications 2006, IEEE Computer Society.

[4] Benhabiles H., Vandeborre J., Lavoué G. et Daoudi M. (2010). A comparative study of existing metrics for 3D-mesh segmentation evaluation. Vis. Comput., vol. 26(12), 14511466.

[5] Benhabiles H., Vandeborre J., Lavoué G. et Daoudi M. (2009). A framework for the objective evaluation of segmentation algorithms using a ground-truth of human segmented 3D models. En SMI : Proceedings of the IEEE International Conference on Shape Modeling and Applications, 36-43. 
[6] Benhabiles H., Vandeborre J., Lavoué G. et Daoudi M. (2009). Une collection de modèles $3 \mathrm{D}$ avec vérité-terrain pour l'évaluation objective des algorithmes de segmentation. En CORESA.

[7] Chen X., Golovinskiy A. et Funkhouser T. (2009). A Benchmark for 3D Mesh Segmentation. ACM Transactions on Graphics (Proc. SIGGRAPH), vol. 28(3).

[8] Delest S., Boné R., et Cardot H. (2008). Réflexions sur la mise en place d’une vérité terrain pour la segmentation de maillages polygonaux 3d en parties significatives. Rapport technique, GTMG : Groupe de Travail en Modélisation Géométrique, 49-62.

[9] Golovinskiy A. et Funkhouser T. (2008). Randomized cuts for 3D mesh analysis. ACM Transactions on Graphics (Proc. SIGGRAPH ASIA), vol. 27(5), 145-157.

[10] Katz S., Leifman G. et Tal A. (2005). Mesh segmentation using feature point and core extraction. Vis. Comput., vol. 21(8), 649-658.

[11] Lai Y.-K., HU S.-M., Martin R. R. and ROSIN P. L. (2008). Fast mesh segmentation using random walks. En ACM Symposium on Solid and Physical Modeling. 183-191.

[12] Lavoué G., Dupont F. et Baskurt A. (2005). A new CAD mesh segmentation method, based on curvature tensor analysis. Computer-Aided Design, vol. 37(10), 975-987.

[13] Meyer M., Desbrun M., Schröder P., et al. (2002). Discrete Differential-Geometry Operators for Triangulated 2-Manifolds. International Workshop on Visualization and Mathematics, 35-57.

[14] Shapira L., Shamir A. et Cohen-Or D. (2008). Consistent mesh partitioning and skeletonisation using the shape diameter function. Vis. Comput., vol. 24(4), 249-259.

[15] Shlafman S., Tal A. et Katz S. (2002). Metamorphosis of polyhedral surfaces using decomposition. Computer Graphics forum, vol. 21(3), 219-228.

[16] Zhang H., Fritts E. J. et Goldman A. S. (2008). Image segmentation evaluation: A survey of unsupervised methods. Comput. Vis. Image Underst., vol. 110 (2), 260-280. 